Article

\title{
A Revised LRSPR Sensor with Sharp Reflection Spectrum
}

\section{Yinquan Yuan * and Yutang Dai}

National Engineering Laboratory for Fiber Optic Sensing Technology, Wuhan University of Technology, Wuhan 430070, China; E-Mail: daiyt6688@whut.edu.cn

* Author to whom correspondence should be addressed; E-Mail: ymyyq@whut.edu.cn; Tel.: +86-027-8765-1850 (ext. 1107); Fax: +86-027-8765-1851.

Received: 18 July 2014; in revised form: 25 August 2014 / Accepted: 1 September 2014 / Published: 5 September 2014

\begin{abstract}
In this work, we have proposed a novel long-range surface plasmon resonance (LRSPR) sensor with sharp reflection spectrum, which consists of a glass prism, a $(\mathrm{A} / \mathrm{B})^{4}$-type waveguide-coupled layer and a metal layer. To reveal its sharp reflection spectrum perfectly, we have simulated the effects of all factors of this LRSPR sensor on the reflection spectrum, and finally presented the optimal parameters of the LRSPR sensor with sharp reflection spectrum.
\end{abstract}

Keywords: optical sensor; surface plasmon resonance; waveguide-coupled; multi-layer model

\section{Introduction}

Surface plasmon resonance (SPR) refers to the optical excitation of surface plasmon wave at the interface between a noble metal and a dielectric. The SPR sensors have great potential in chemical and biological sensing, including the measurements on the concentration of an analyte in a complex sample, the specificity, affinity and biomolecular interaction kinetics [1-3]. The most common SPR sensor, also called the conventional SPR (CSPR) sensor, is in Kretschmann configuration and comprises of a glass prism, a thin metal film and a sample in sequence, that may work in angular or wavelength interrogation mode. For the SPR sensor in angular interrogation mode, the wavelength of the entrance light is constant and the incident angle can be adjusted [4-6]. Until now, numerous works have been done to develop the CSPR sensors in angular interrogation mode [7-11], but these CSPR sensors have a common disadvantage, namely the wide reflection spectrum $\left(\sim 2-3^{\circ}\right.$ of dip width $)$ that produces low sign-to-noise ratio. 
Apart from CSPR sensors, other three kinds of SPR sensors have been developed also [12]. The first kind is waveguide-coupled SPR (WCSPR) sensor, whose configuration is made up of a prism, a metal layer, a dielectric waveguiding layer, a metal layer and a sample in sequence [13-15]. In the reflection spectrum of WCSPR sensor, there are one CSPR dip, one WCSPR dip and several waveguide-coupled resonance dips. Although the WCSPR dip is one sharp dip $\left(\sim 0.3^{\circ}\right)$, it is difficult to distinguish those dips in the measurements. The second kind is plasmon-waveguide SPR (PWSPR) sensor, whose configuration is made up of a prism, a metal layer, a dielectric waveguiding layer and a sample in sequence $[16,17]$. In its reflection spectrum, there are one CSPR dip and one sharp WCSPR $\operatorname{dip}\left(\sim 0.5^{\circ}\right)$, but our simulations have shown that the refractive index (RI) range of the WCSPR dip is too small.

The third kind is a long-range SPR (LRSPR) sensor, whose configuration is made up of a prism, a dielectric layer, a metal layer and a sample in sequence [18-21]. Based on LRSPR structure, we have proposed a revised LRSPR sensor with sharp reflection spectrum, which consists of a glass prism, a $(\mathrm{A} / \mathrm{B})^{4}$-type waveguide-coupled (WC) layer and a metal layer. To reveal its sharp reflection spectrum perfectly, we have investigated the effects of key parameters of the revised LRSPR sensor on the reflection spectrum, and finally presented the optimal parameters.

\section{Model and Simulation Results}

Figure 1a gives the configuration of the revised LRSPR sensor. The first layer is the coupling prism with the RI of $n_{\mathrm{p}}=1.5105$. As shown in Figure $1 \mathrm{~b}$, the next is the WC layer with the periodic structure of type $(\mathrm{A} / \mathrm{B})^{Y}$, where $\mathrm{A}=\mathrm{TiO}_{2}$ and $\mathrm{B}=\mathrm{SiO}_{2}, Y$ denotes the number of A/B layer, the $\mathrm{RI}$ and thickness of A layer is denoted by $n_{2}(=2.232)$ and $d_{2}$, the RI and thickness of B layer denoted by $n_{3}(=1.451)$ and $d_{3}$ respectively. Let $N=2 Y+3$, then the $(N-1)$ th layer is the noble metal layer whose dielectric function obeys Lorentz-Drude model and can be expressed in the following form [22]

$$
\varepsilon_{\mathrm{m}}(\omega)=1-\frac{f_{0} \omega_{\mathrm{p}}^{2}}{\omega\left(\omega+i \Gamma_{0}\right)}+\sum_{j=1}^{k} \frac{f_{j} \omega_{\mathrm{p}}^{2}}{\omega_{j}^{2}-\omega^{2}+i \omega \Gamma_{0}}
$$

where $\omega=2 \pi \mathrm{c} / \lambda$ is the light frequency, $\omega_{\mathrm{p}}$ the plasma frequency, $k$ the number of oscillators with frequency $\omega_{j}$, strength $f_{j}$, and lifetime $1 / \Gamma_{j}$, while $f_{0} \omega_{\mathrm{p}}{ }^{2}$ is the plasma frequency associated with intraband transitions with oscillator strength $f_{0}$ and damping constant $\Gamma_{0}$. The $N$ th layer is the sample with the RI denoted by $n_{\text {sam. }}$.

To calculate the reflected light intensity of $p$-polarized incident light, the $N$-layer model has been used to simulate the SPR sensor working in the angle interrogation mode. As shown in Figure 2, these layers are stacked along the $z$-axis, any layer is defined by the thickness $d_{\mathrm{k}}$, dielectric constant $\varepsilon_{\mathrm{k}}$, and refractive index $n_{\mathrm{k}}$, where $k$ is a footnote that denotes the prism, metal, dielectric layers and the sample. All layers are assumed to be uniform, isotropic and non-magnetic. The tangential fields at the first boundary $Z=Z_{1}=0$ are related to those at the final boundary $Z=Z_{N-1}$ by $[3,22]$

$$
\left[\begin{array}{c}
U_{1} \\
V_{1}
\end{array}\right]=M\left[\begin{array}{l}
U_{N-1} \\
V_{N-1}
\end{array}\right]
$$


where $U_{1}$ and $V_{1}$, respectively, are the tangential components of electric and magnetic fields at the boundary of first layer, $U_{N-1}$ and $V_{N-1}$ are the corresponding fields at the boundary of $N$ th layer, $M$ is known as characteristic matrix of the combined structure and is given by

Figure 1. Configuration of the revised long-range surface plasmon resonance (LRSPR) sensor.

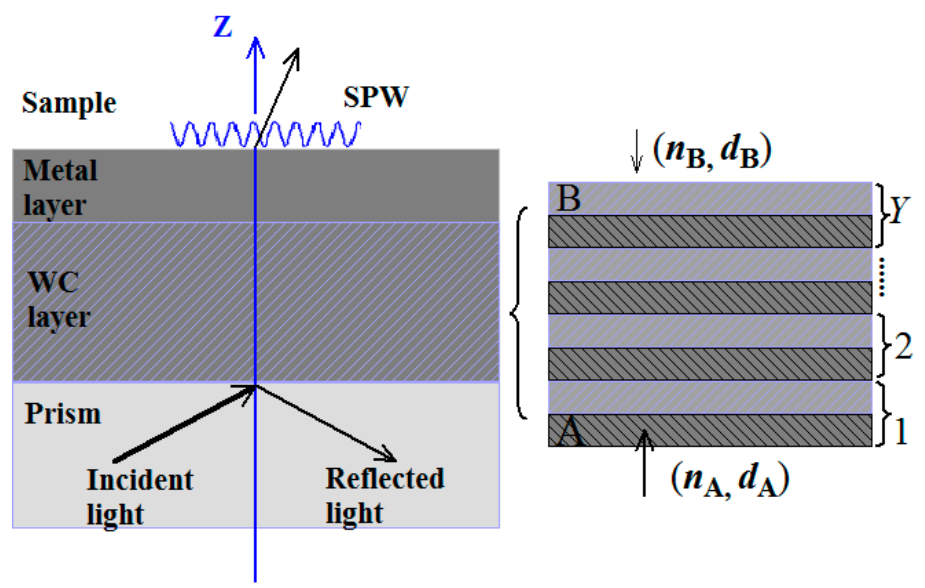

Figure 2. Multi-layer model of the surface plasmon resonance (SPR) sensor.

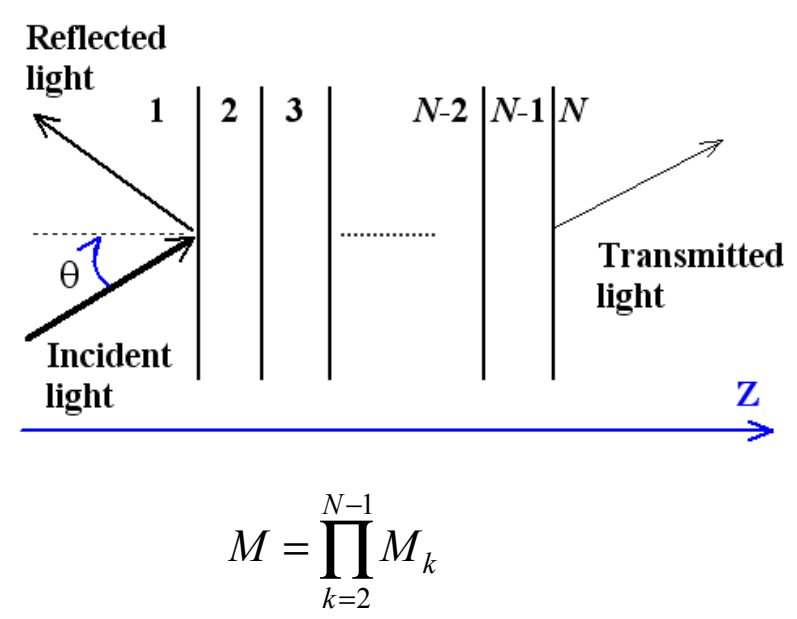

with

$$
M_{k}=\left[\begin{array}{cc}
\cos \beta_{k} & -\mathrm{i} \sin \beta_{k} / q_{k} \\
-\mathrm{i} q_{k} \sin \beta_{k} & \cos \beta_{k}
\end{array}\right]
$$

where

$$
\begin{gathered}
q_{k}=\frac{\left(\varepsilon_{k}-n_{1}^{2} \sin ^{2} \theta_{1}\right)^{1 / 2}}{\varepsilon_{k}} \\
\beta_{k}=\frac{2 \pi d_{k}}{\lambda}\left(\varepsilon_{k}-n_{1}^{2} \sin ^{2} \theta_{1}\right)^{1 / 2}
\end{gathered}
$$

Here $\lambda$ is the free space wavelength. The amplitude reflection coefficient for $p$-polarized incident wave is given by 


$$
r_{\mathrm{p}}=\frac{\left(M_{11}+M_{12} q_{N}\right) q_{1}-\left(M_{21}+M_{22} q_{N}\right)}{\left(M_{11}+M_{12} q_{N}\right) q_{1}+\left(M_{21}+M_{22} q_{N}\right)}
$$

Finally, the reflection intensity for $p$-polarized light is

$$
R_{\mathrm{p}}=\left|r_{\mathrm{p}}\right|^{2}
$$

Firstly, to understand the effect of the number of A/B layers on the SPR, Figure 3 depicts the reflection spectra of the revised LRSPR sensors with different number of A/B layers (1, 2, 3 and 4), where the RI $n_{\text {sam }}=1.3330$, the thicknesses $d_{2}=100 \mathrm{~nm}$ and $d_{3}=400 \mathrm{~nm}$ were chosen. It can be seen that, the LRSPR sensor with one A/B layer has flat resonance spectrum (full width at half minimum, FWHM $\left.\Delta \theta_{\mathrm{FWHM}}=1.81^{\circ}\right)$, the sensor with three $\mathrm{A} / \mathrm{B}$ layers has a better resonance dip $\left(\Delta \theta_{\mathrm{FWHM}}=0.30^{\circ}\right)$ and the sensor with four A/B layers has a sharpest resonance $\operatorname{dip}\left(\Delta \theta_{\mathrm{FWHM}}=0.19^{\circ}\right)$. We have also calculated the reflection spectra of the LRSPR sensors with five and more A/B layers, and found that their reflection spectra have no obvious improvement. In addition, the increase of A/B layers causes the preparation difficulty of the LRSPR sensor, so we take $Y=4$ in the following discussion.

Figure 3. Reflection spectra of the revised LRSPR sensors with different numbers of A/B layers.

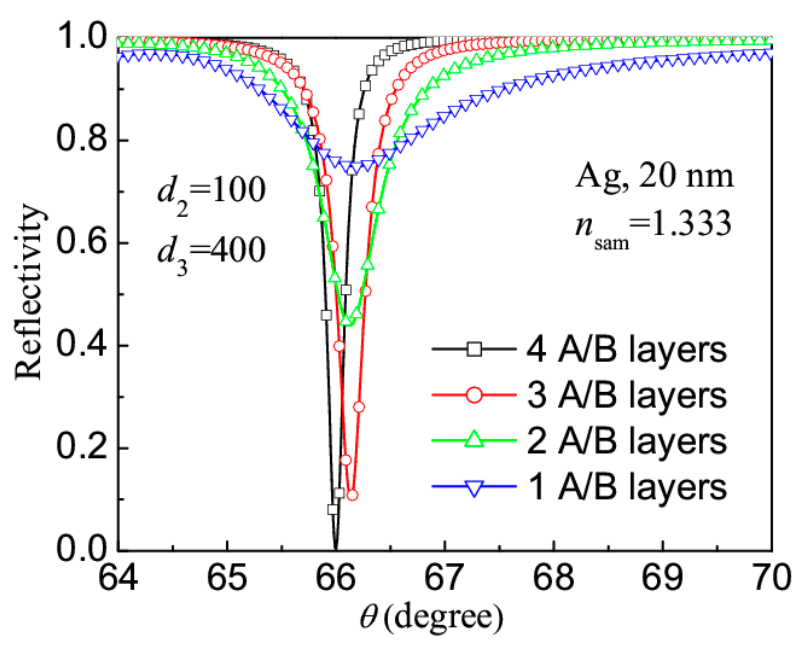

Then we have investigated the effects of different metals and thicknesses for the LRSPR sensor with 4 A/B layers. Figure 4 gives the reflection spectra of the sensors corresponding to metals $\mathrm{Ag}$ and Au with different thicknesses, where $n_{\mathrm{sam}}=1.333, d_{2}=80 \mathrm{~nm}$ and $d_{3}=400 \mathrm{~nm}$ were chosen. For the sensors with Ag layer (Figure 4a), when the thickness of Ag layer changes from $15 \mathrm{~nm}$ to $30 \mathrm{~nm}$, the resonance angle $\left(\theta_{\text {res }}\right)$ increases in the range of $64.9-66.3^{\circ}$, all the resonance dips are very sharp, and the best choice is the Ag layer of $20 \mathrm{~nm}$. When using Au to prepare the metal layer of the sensor (Figure $4 \mathrm{~b})$, the resonance dips are wider, the signal-to-noise ratio $\left(\Delta \theta_{\mathrm{res}} / \Delta \theta_{\mathrm{FWHM}}\right)$ is lower, and it is recommended that its thickness should not be greater than $20 \mathrm{~nm}$.

Coming from above discussions, better reflection spectra can be obtained by using the Ag layer of $20 \mathrm{~nm}$, so such a metal layer was fixed in the following. Figure 5 shows the effects of the thicknesses $d_{2}$ and $d_{3}$ on the reflection spectrum of the revised LRSPR sensor, where $n_{\text {sam }}=1.333 \mathrm{~nm}, d_{3}=400 \mathrm{~nm}$ 
(a) or $d_{2}=100 \mathrm{~nm}$ (b) were fixed. It can be seen that, when $d_{2}$ is in the range of 70-120 nm and $d_{3}$ in the range of 300-450 $\mathrm{nm}$, the reflection spectrum has better resonance dip.

Finally, to understand the sensitivity $\left(\Delta \theta_{\text {res }} / \Delta n_{\text {sam }}\right)$ and signal-to-noise ratio $\left(\Delta \theta_{\text {res }} / \Delta \theta_{\text {FWHM }}\right)$ of the revised LRSPR sensor, Figure 6 shows the effect of sample RI on the reflection spectrum of the revised LRSPR sensor, where $d_{2}=100 \mathrm{~nm}$ and $d_{3}=400 \mathrm{~nm}$ were fixed. When the sample RI increases from 1.330 to 1.400 , the resonance angle increases linearly from $65.85^{\circ}$ to $70.05^{\circ}$, the sensitivity $\Delta \theta_{\text {res }} / \Delta n_{\text {sam }}$ is $60^{\circ} /$ RIU approximately. Moreover, their spectrum width $\left(\Delta \theta_{\text {FWHM }}<0.2^{\circ}\right)$ is smaller than one 10th of the spectrum width $\left(\Delta \theta_{\mathrm{FWHM}}>2^{\circ}\right)$ of the CSPR sensor, so their signal-to-noise ratio ( $\Delta \theta_{\text {res }} / \Delta \theta_{\text {FWHM }}$ ) is 10 times greater than that of the CSPR sensor.

Figure 4. Reflection spectra of the revised LRSPR sensors with Ag or Au layer.
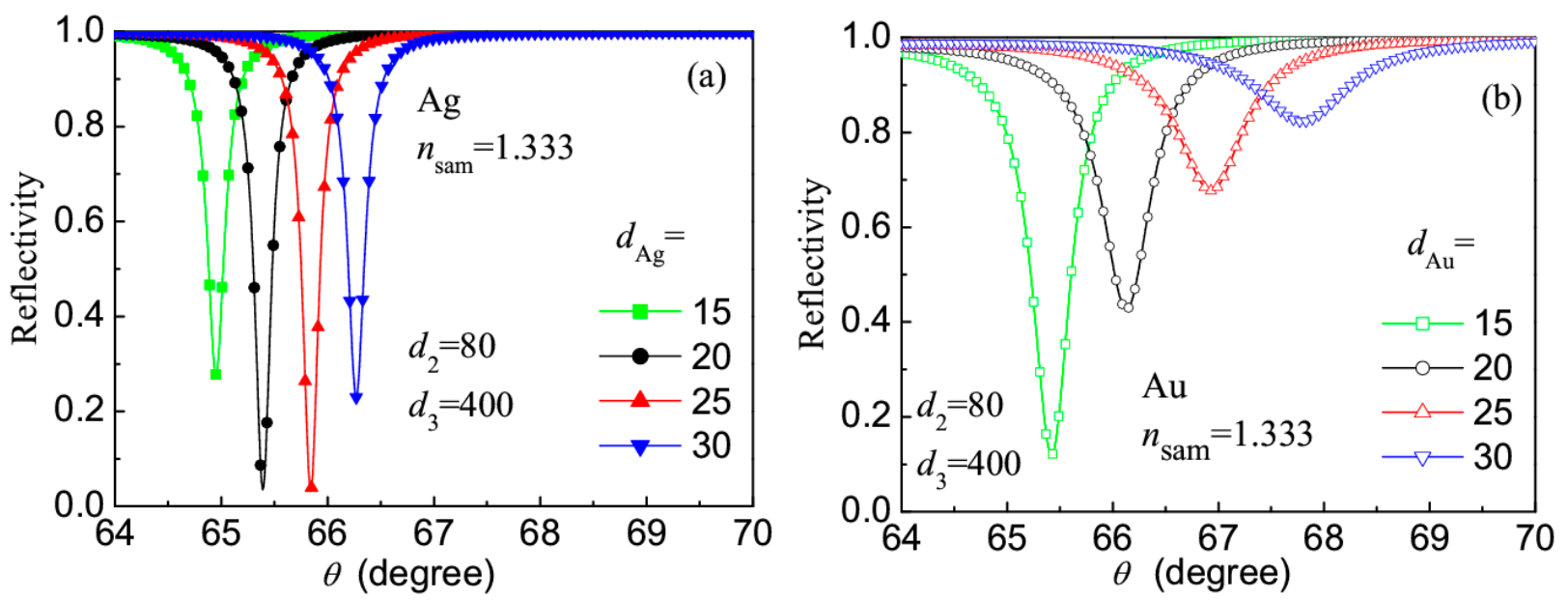

Figure 5. Effects of thicknesses $d_{2}$ and $d_{3}$ on the reflection spectrum of the revised LRSPR sensor.
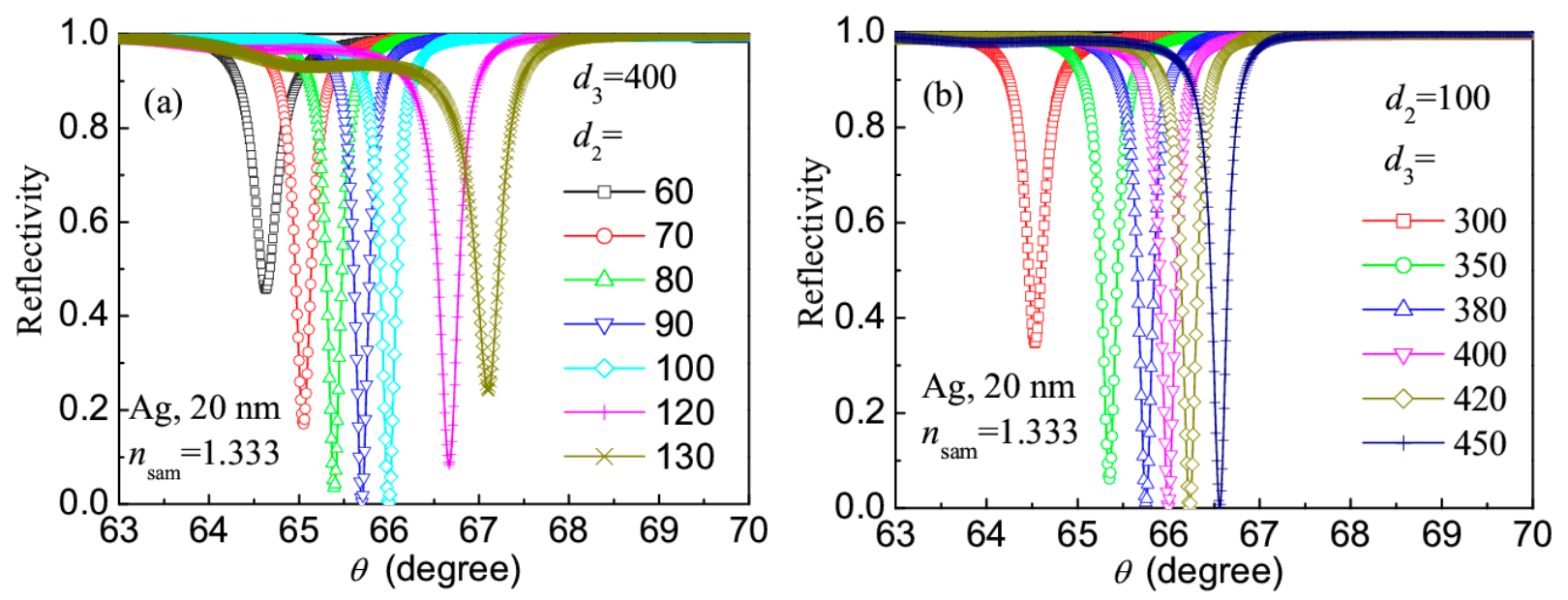
Figure 6. Reflection spectra of the revised LRSPR sensor corresponding to different sample RIs.

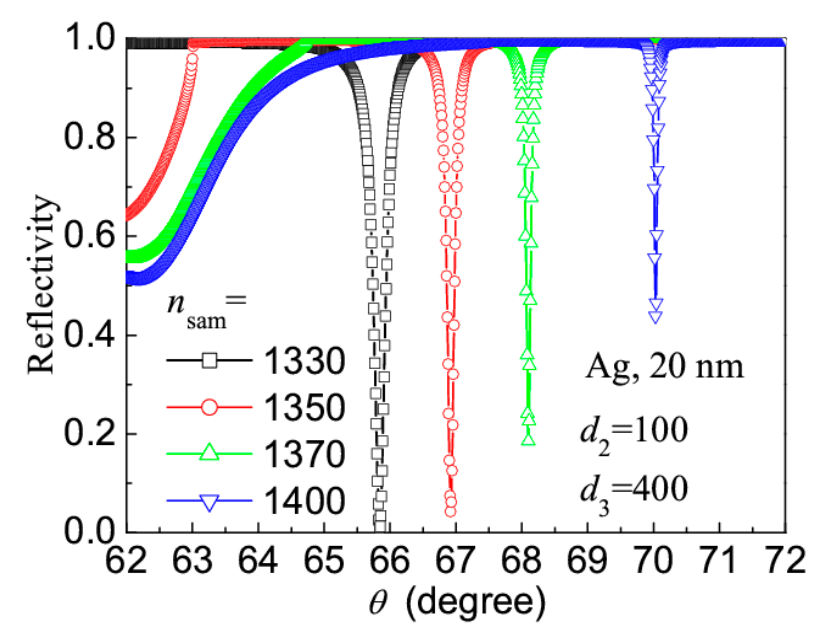

\section{Conclusions}

We have proposed a revised LRSPR sensor with sharp reflection spectrum, it consists of a glass prism, a $(\mathrm{A} / \mathrm{B})^{4}$-type waveguide-coupled layer and a silver layer. Using the Ag layer with the thickness of about $20 \mathrm{~nm}$, when the thickness of A layer $\left(\mathrm{TiO}_{2}, \mathrm{RI}=2.232\right)$ is in the range of $70-120 \mathrm{~nm}$ and the thickness of $\mathrm{B}$ layer $\left(\mathrm{SiO}_{2}, \mathrm{RI}=1.451\right)$ is in the range of $300-450 \mathrm{~nm}$, the reflection spectrum of the revised LRSPR sensor has a better resonance dip with the FWHM of about $0.2^{\circ}$, which is smaller than one 10th of the spectrum width of the CSPR sensor. Moreover, when the sample RI increases from 1.330 to 1.400 , the resonance angle increases linearly from $65.85^{\circ}$ to $70.05^{\circ}$ and the sensitivity of $60 \%$ RIU was obtained.

\section{Acknowledgments}

This work is finically supported by the Project of National Science Foundation of China (No. 61475121, 50802069).

\section{Author Contributions}

Yutang Dai finished parts of figure drawing and paper writing; Yinquan Yuan finished the establishing of theoretical model, the simulations and discussions, and other works.

\section{Conflict of Interest}

The authors declare no conflict of interest.

\section{References}

1. Homola, J. Present and future of surface plasmon resonance biosensors. Anal. Bioanal. Chem. 2003, 377, 528-539.

2. Hoa, X.D.; Kirk, A.G.; Tabrizian, M. Towards integrated and sensitive surface plasmon resonance biosensors: A review of recent progress. Biosens. Bioelectron. 2007, 23, 151-160. 
3. Gupta, G.; Kondoh, J. Tuning and sensitivity enhancement of surface plasmon resonance sensor. Sens. Actuators B Chem. 2007, 122, 381-388.

4. Agbor, N.E.; Cresswell, J.P.; Petty, M.C.; Monkman, A.P. An optical gas sensor based on polyaniline Langmuir-Blodgett films. Sens. Actuators B Chem. 1997, 41, 137-141.

5. Chien, F.C.; Chen, S.J. A sensitivity comparison of optical biosensors based on four different surface plasmon resonance modes. Biosens. Bioelectron. 2004, 20, 633-642.

6. Morjana, M.; Züchnerb, H.; Cammann, K. Contributions to a reliable hydrogen sensor based on surface plasmon surface resonance spectroscopy. Surf. Sci. 2009, 603, 1353-1359.

7. Fernandez, C.J.; Manera, M.G.; Pellegrini, G.; Bersani, M.; Mattei, G.; Rella, R.; Vasanelli, L.; Mazzoldi, P. Surface plasmon resonance optical gas sensing of nanostructured ZnO films. Sens. Actuators B Chem. 2008, 130, 531-537.

8. Piliarik, M.; Bockova, M.; Homola, J. Surface plasmon resonance biosensor for parallelized detection of protein biomarkers in diluted blood plasma. Biosens. Bioelectron. 2010, 26, 1656-1661.

9. Hooper, I.R.; Rooth, M.; Sambles, J.R. Dual-channel differential surface plasmon ellipsometry for bio-chemical sensing. Biosens. Bioelectron. 2009, 25, 411-417.

10. El-Basaty, A.B.; El-Brolossy, T.A.; Abdalla, S.; Negm, S.; Abdella, R.A.; Talaat, H. Surface plasmon sensor for $\mathrm{NO}_{2}$ gas. Surf. Interface Anal. 2008, 40, 1623-1626.

11. Bolduc, O.R.; Live, L.S.; Masson, J.F. High-resolution surface plasmon resonance sensors based on a dove prism. Talanta 2009, 77, 1680-1687.

12. Abbas, A.; Linman, M.J.; Cheng, Q. New trends in instrumental design for surface plasmon resonance-based biosensors. Biosens. Bioelectron. 2011, 26, 1815-1824.

13. Kovacs, G.J.; Scott, G.D. Optical excitation of surface plasma waves in layered media. Phys. Rev. B 1977, 16, 1297-1311.

14. Wang, K.; Wan, Y.; Zheng, Z.; He, F.; Hu, Z.; Zhu, J. A novel refractive index detection method in voltage scanning surface plasmon resonance system. Sens. Actuators B Chem. 2012, 169, 393-396.

15. Ma, X.; Xu, X.; Zheng, Z.; Wang, K.; Su, Y.; Fan, J.; Zhang, R.; Song, L.; Wang, Z.; Zhu, J. Dynamically modulated intensity interrogation scheme using waveguide coupled surface plasmon resonance sensors. Sens. Actuators A Phys. 2010, 157, 9-14.

16. Salamon, Z.; Macleod, H.A.; Tollin, G. Coupled plasmon-waveguide resonators: A new spectroscopic tool for probing proteolipid film structure and properties. Biophys. J. 1997, 73, 2791-2797.

17. Lau, K.H.A.; Tan, L.S.; Tamada, K.; Sander, M.S.; Knoll, W. Highly sensitive detection of processes occurring inside nanoporous anodic alumina templates: A waveguide optical study. J. Phys. Chem. B 2004, 108, 10812-10818.

18. Sarid, D. Long-range surface-plasma waves on very thin metal films. Phys. Rev. Lett. 1981, 47, 1927-1930.

19. Quail, J.C.; Rako, J.G.; Simon, H.J. Long-range surface plasmon modes in silver and aluminum films. Opt. Lett. 1983, 8, 377-379.

20. Nenninger, G.G.; Tobisk, P.; Homola, J. Long-range surface plasmons for high-resolution surface plasmon resonance sensors. Sens. Actuators B Chem. 2001, 74, 145-151. 
21. Slavik, R.; Homola, J. Ultrahigh resolution long range surface plasmon-based sensor. Sens. Actuators B Chem. 2007, 123, 10-12.

22. Yuan, Y.; Ding L.; Guo, Z. Numerical investigation for SPR-based optical fiber sensor. Sens. Actuators B Chem. 2011, 157, 240-245.

(C) 2014 by the authors; licensee MDPI, Basel, Switzerland. This article is an open access article distributed under the terms and conditions of the Creative Commons Attribution license (http://creativecommons.org/licenses/by/3.0/). 